\title{
Efecto del nivel de urea del sacchapulido sobre la degradación del forraje elefante (Pennisetum purpureum)
}

\section{Effect of the urea level of sacchapulido on the degradation of elephant grass (Pennisetum purpureum)}

\author{
Jesús Alberto Ramos-Juárez ${ }^{1}$, Luis Manuel Vargas-Villamil ${ }^{1 *}$, Francisco Izquierdo-Reyes ${ }^{1}$, Carlos Rubén \\ Fernández-Cabrera ${ }^{1}$, Germán David Mendoza-Martínez ${ }^{2}$, Emilio Manuel Aranda-Ibañez ${ }^{1}$, David \\ Hernández-Sánchez ${ }^{3}$, Arabel Elías-Iglesias ${ }^{4}$ \\ ${ }^{1}$ Maestría en Producción Agroalimentaria en el Trópico, Colegio de Postgraduados, Campus Tabasco, Periférico Carlos A. \\ Molina, Km 3.5. Carretera Cárdenas-Huimanguillo, CP. 86500. H. Cárdenas, Tabasco, México. \\ ${ }^{2}$ Universidad Autónoma Metropolitana, Unidad Xochimilco, Departamento de Producción Agrícola y Animal, Calzada del \\ Hueso 1100, Colonia Villa Quietud, CP. 04960. Ciudad de México, México. \\ ${ }^{3}$ Colegio de Postgraduados, Campus Montecillo. Carretera México-Texcoco Km. 36.5, CP. 56230. Montecillo, Texcoco, Estado \\ de México, México. \\ ${ }^{4}$ Instituto de Ciencia Animal. Carretera Central Km 47.5, San José de las Lajas, CP. 32700. La Habana, Cuba. \\ ${ }^{*}$ Autor de correspondencia: luis@avanzavet.com
}

Nota científica recibido: 18 de julio de 2017 aceptado: 06 de marzo de 2018

RESUMEN. Se evaluó el efecto del nivel de urea sobre la degradación del pasto Elefante (Pennisetum purpureum) en el suplemento fermentado, Sacchapulido (SP). Se determinó el pH, N-NH 3 , la degradación in situ (DI) y efectiva (DE), así como, los parámetros cinéticos de materia seca (MS), FDN y FDA del forraje. Se utilizaron cinco bovinos fistulados en rumen en un diseño cuadro Latino $5 \times 5$. La DE indicó que los suplementos que tienen entre 0.5 y $1.0 \%$ de urea, aportaron un $10 \%$ más de biomasa por el aumento de tamaño de la fracción potencialmente degradable (PD) del alimento, tanto en la FDN, como en el contenido celular. Debido al costo de la urea, se recomienda el uso del SP con $0.5 \%$ de urea.

Palabras clave: Fermentación ruminal, fracción de fibra, caña de azúcar, fermentación en estado sólido

ABSTRACT. The effect of the urea level of the fermented supplement Sacchapulido (SP) on the degradation of elephant grass (Pennisetum purpureum) was evaluated. The $\mathrm{pH}, \mathrm{N}-\mathrm{NH}_{3}$ and in situ and effective degradation (ID and ED respectively) were determined, as well as the kinetic parameters of dry matter (DM), NDF and ADF of the forage. Five fistulated bovines were used in a $5 \times 5$ Latin square design. The standard deviation indicated that the supplements have between 0.5 and $1.0 \%$ urea; they contributed $10 \%$ more biomass due to the increase in size of the potentially degradable fraction of the feed, both in the NDF and cellular content. Due to the cost of urea, the use of SP with $0.5 \%$ urea is recommended.

Key words: Ruminal fermentation, fiber fraction, sugarcane, solid-state fermentation

\section{INTRODUCCIÓN}

Los forrajes son la base de la alimentación en los sistemas de producción bovina en el trópico (Cruz-Hernández et al. 2017). Pero presentan deficiencias nutricionales, como baja digestibilidad de la MS (55\%) y reducido contenido de proteína $(7.7 \%)$, lo que limita la actividad microbiana en el rumen, el consumo voluntario y la producción de carne $(0.45$ $\mathrm{kg} \mathrm{d}^{-1}$ ) o leche (1400 kg por lactancia) (Corea et al. 2017). Por lo que, requieren suplementos para complementar las deficiencias de nutrientes de los pastos (Reid et al. 2015).

El Sacchapulido (SP) es un concentrado energético-proteínico obtenido de la caña de azúcar por fermentación en estado sólido (FES), el cual es 
de fácil elaboración con ingredientes de la misma finca (Ramos et al. 2016). El SP tiene un efecto positivo en la degradación ruminal del forraje y en las condiciones para incrementar el crecimiento microbiano, manteniendo el $\mathrm{pH}$ ruminal en niveles que no afectan la celulolísis. Su importancia radica en su capacidad de aportar nitrógeno amoniacal (N$\mathrm{NH}_{3}$ ), carbohidratos solubles, proteína verdadera, minerales, aminoácidos, AGV, enzimas y otros elementos a la dieta (Sosa et al. 2016).

El tamaño y tipo de población microbiana determina la velocidad, cantidad de material degradado de MS y sus diferentes fracciones (Galindo et al. 1995). Pero requiere de la interacción del nitrógeno con la energía para la degradación de la materia seca (MS) (Uddin et al. 2015). Esta interacción es diferente para cada tipo de suplemento, por lo que es importante estudiar el efecto de las concentraciones del nitrógeno (N) sobre la degradación, tanto para conocer el potencial nutritivo del suplemento, así como, para tener criterios que mejoren el aprovechamiento de la MS (Galindo et al. 1995). Por lo anterior el objetivo del presente trabajo fue evaluar el efecto de los niveles de urea en el SP sobre la degradación ruminal de la MS y de las fracciones de fibra en dietas a base pasto elefante (Pennisetum purpureum).

\section{MATERIALES Y MÉTODOS}

La investigación se realizó en el Campus Tabasco del Colegio de Postgraduados, México. El sitio se localiza a los $18^{\circ} 00^{\prime} \mathrm{LN}$ y $93^{\circ} 30^{\prime} \mathrm{LO}$, a una altitud de $9 \mathrm{msnm}$, con clima tropical húmedo; con temperatura y precipitación media anual de $26.2{ }^{\circ} \mathrm{C}$ y $2240 \mathrm{~mL}$, respectivamente. Se utilizaron cinco bovinos cruzados (Bos tauros $x$ Bos indicus) de $490.6 \pm 15.08 \mathrm{~kg}$ de peso vivo, canulados en rumen y alojados en corrales individuales con agua y sales minerales a voluntad. Los tratamientos evaluados fueron: Solo forraje $(\mathrm{sF}), \mathrm{sF}+\mathrm{SP}$ con $0.5 \%$ de urea, $\mathrm{sF}+\mathrm{SP}$ con $1.0 \%$ urea, sF + SP con $1.5 \%$ urea y $\mathrm{sF}+\mathrm{SP}$ con $2.0 \%$ de urea.

El forraje Elefante (Pennisetum purpureum
Schumacher) fue ofrecido a libre acceso, picado en un molino tipo Chetumal, a las $8: 00$ y $14: 00$ $\mathrm{h}$, permitiendo un rechazo mínimo del $20 \%$ de lo ofrecido el día anterior. El SP se les ofreció a las 8:00 h a razón de $6 \mathrm{~g} \mathrm{~kg}^{-1}$ de peso vivo. Se utilizó tallo de caña de azúcar limpia (sin hojas, sin pajas y sin cogollo), que se molió en una picadora de forraje a las $24 \mathrm{~h}$ de cortada y se mezcló con los ingredientes según tratamientos (Tabla 1 ). Una vez mezclados, los alimentos se extendieron en una superficie de concreto, libre de los rayos solares, con un espesor de $10 \mathrm{~cm}$, fermentándose por $24 \mathrm{~h}$, y almacenándose en bolsas de polietileno. Los alimentos se elaboraron cada siete días. Los animales tuvieron $10 \mathrm{~d}$ de adaptación al suplemento y cuatro días de muestreo.

El líquido ruminal de los toretes se colectó en el saco ventral a las $0,2,4,6,12$ y 24 h después de suministrar el suplemento correspondiente, y se midió el pH con un potenciómetro digital (Denver Instrument $^{\mathrm{TM}} \mathrm{pH} / \mathrm{mV}$ modelo UB-10) por cuatro días. Posteriormente, $4 \mathrm{~mL}$ del líquido ruminal fueron acidificados con $1 \mathrm{~mL}$ de ácido metafosfórico al $25 \%$ y se almacenaron a baja temperatura para el análisis posterior de nitrógeno amoniacal ( $\mathrm{N}$ $\mathrm{NH}_{3}$ ), según Pardo et al. (2008), el cual se determinó en un espectofotómetro de luz ultravioleta visible CARY 1-E UARIAN con longitud de onda $630 \mathrm{~nm}$ sin columna. La degradación in situ de la MS (DIMS), FDN (DIFDN) y FDA (DIFDA) del forraje, en cada recolección del líquido ruminal, se determinó incubando por duplicado $7 \mathrm{~g}$ de forraje en el rumen por 2, 4, 6, 12, 24, 48, 72 y $96 \mathrm{~h}$ en bolsas de tela nylon $(10 \times 20 \mathrm{~cm}$, porosidad 45 $\mu \mathrm{m}$ ), con la técnica de la bolsa de nylon (Haile et al. 2017), después de la suplementación con SP. Posteriormente, se mezclaron los residuales de la MS para cada tratamiento y tiempo de incubación, y se obtuvieron dos muestras para la evaluación de la DIFDN y la DIFDA. Posteriormente, se secaron en una estufa de aire forzado a $62{ }^{\circ} \mathrm{C}$ por $48 \mathrm{~h}$, calculando la biomasa por diferencia de peso. La determinación del contenido de MS, materia orgánica (MO), cenizas, proteína bruta (PB), nitrógeno no 
Tabla 1. Ingredientes (base fresca) usados para elaborar el Sacchapulido con diferentes niveles de urea $(\mathrm{U})$.

\begin{tabular}{lcccc}
\hline \multirow{2}{*}{ Ingredientes, \% } & \multicolumn{4}{c}{ Nivel de urea } \\
\cline { 2 - 5 } & $0.5 \%$ & $1.0 \%$ & $1.5 \%$ & $2.0 \%$ \\
\hline Caña de azúcar & 74.70 & 74.20 & 73.70 & 73.20 \\
Pasta de soya & 4.00 & 4.00 & 4.00 & 4.00 \\
Pulido de arroz & 20.00 & 20.00 & 20.00 & 20.00 \\
Urea, $\mathrm{CO}\left(\mathrm{NH}_{2}\right)_{2}$ & 0.50 & 1.00 & 1.50 & 2.00 \\
Sulfato de amonio, $\left(\mathrm{NH}_{4}\right)_{2} \mathrm{SO}_{4}$ & 0.30 & 0.30 & 0.30 & 0.30 \\
Minerales & 0.50 & 0.50 & 0.50 & 0.50 \\
\hline
\end{tabular}

proteínico (NNP) (AOAC 2016); mientras que el contenido de proteína bruta (PB) se determinó de acuerdo con Kirk et al. (2011); en tanto que el contenido de proteína verdadera (PV), se calculó por diferencia con la siguiente formula PV $=$ PB - (NNP*6.25); mientras que el contenido de FDN, FDA y contenido celular (CC), se calculó por diferencia como $C C=100$ - FDA de acuerdo con (Lourenço et al. 2017).

Para la evaluación estadística del $\mathrm{pH}, \mathrm{N}-\mathrm{NH}_{3}$ y DIMS, los cinco tratamientos se alojaron en un cuadrado Latino $(5 \times 5)$ con medidas repetidas, donde los períodos constituyeron las hileras, y las columnas los cinco animales, incluyendo como factor dentro de sujetos al tiempo de incubación. En la DIFDN y DIFDA no se incluyó el factor periodos. Se realizó un análisis de varianza de acuerdo al diseño experimental propuesto y con el siguiente modelo lineal mixto: $Y_{i j k l}=\mu+\alpha_{i}+\rho_{j}+\tau_{k(i, j)}+\gamma_{l}+\pi_{k l}+$ $\theta_{i j k}+\varepsilon_{i j k l}$; para $\mathrm{i}=1,2, \ldots, 5 ; \mathrm{j}=1,2, \ldots, 5 ; \mathrm{k}=1$, $2, \ldots, 5 ; I=1,2, \ldots, 6$. Donde $Y_{i j k l}$ es la respuesta observada; $\mu$ la media general; $\alpha_{i}$ el efecto aleatorio del i-ésimo animal; $\rho_{j}$ el efecto aleatorio del j-ésimo periodo; $\tau_{k(i, j)}$ el efecto del k-ésimo tratamiento; $\gamma_{l}$ el efecto del l-ésimo tiempo de incubación; $\pi_{k l}$ el efecto de la interacción tratamiento por tiempo de incubación; $\theta_{i j k}$ el efecto aleatorio de la interacción entre animal i, periodo j y el tratamiento k; y $\varepsilon_{i j k l}$ el error aleatorio. Posteriormente, para la interacción tiempo de incubación por tratamientos y efectos principales que fueron significativos $(p<0.05)$, se realizó una prueba de comparación múltiple de medias mediante el método de Tukey (Montgomery 2012), con el software SAS versión 9.4 (SAS 2017).

Los resultados obtenidos de la DI se ajustaron a un modelo dinámico, mecanístico y determinístico para la estimación del sustrato potencialmente degradado (PD) y la tasa de degradación $(k d)$, el cual fue utilizado para la simulación de la degradación efectiva (DE). El modelo consistió en un sistema de tres ecuaciones diferenciales que describen la degradación de los substratos en el rumen por las bacterias en función del material potencialmente degradable (PD) y no degradable (ND). El sistema de ecuaciones diferenciales y auxiliares fueron $\mathrm{dPD} / \mathrm{dt}=-$ Degradación; $\mathrm{dD} / \mathrm{dt}=$ Degradación; $\mathrm{dDE} / \mathrm{dt}=$ Degradación; Degradación $=k d \mathrm{PD}$; Pasaje $=k p P D$; y $\mathrm{R}=\mathrm{PD}+\mathrm{ND}$. Dónde: $\mathrm{D}=$ Sustrato degradado; $\mathrm{DE}=$ Degradación efectiva; $\mathrm{PD}=$ Sustrato potencialmente degradable; ND $=$ Sustrato no degradable; $k d=$ Tasa de degradación; $k p=$ Tasa de pasaje; $\mathrm{R}=$ Residual. El modelo se ajustó a los residuales ( $R$ ) obtenidos experimentalmente para cada tratamiento, utilizando el programa Berkeley Madonna 8.0.1 (Macey et al. 2003) y los parámetros $k d$, PD y ND. Finalmente, se corrieron los modelos con Berkeley Madonna 8.0.1 y tasa de pasaje de 0.03 para obtener la DE, a 1000 $\mathrm{h}$ de simulación.

\section{RESULTADOS Y DISCUSIÓN}

Los valores bromatológicos (Tabla 2) del tratamiento $\mathrm{sF}$ fueron similares a lo reportado por Rusdy (2016). Pero los valores de MS, PB y PV fueron mayores para los tratamientos suplementos con diferentes niveles de urea $(\mathrm{SF}+\mathrm{SP})$, asociándose este incremento a la fermentación al aire libre y no en frascos Roux en laboratorio. Mientras que el contenido de FDN y FDA, son menores a 
Tabla 2. Composición bromatológica del forraje Elefante y del Sacchapulido (SP) con diferentes niveles de urea $(\mathrm{U})$.

\begin{tabular}{lccccc}
\hline \multirow{2}{*}{ Factor (\%) } & \multicolumn{5}{c}{ Tratamientos } \\
\cline { 2 - 6 } & Forraje & SP con $0.5 \%$ U & SP con $1.0 \%$ U & SP con 1.5\% U & SP con $2.0 \%$ U \\
\hline MS & $20.34 \pm 1.7$ & $44.65 \pm 1.1$ & $43.39 \pm 1.4$ & $42.88 \pm 0.3$ & $41.78 \pm 0.7$ \\
Cenizas & $10.25 \pm 0.9$ & $8.18 \pm 1.5$ & $9.19 \pm 3.1$ & $8.65 \pm 1.8$ & $8.79 \pm 1.8$ \\
MO & $89.75 \pm 0.9$ & $91.82 \pm 1.5$ & $90.81 \pm 3.1$ & $91.35 \pm 1.8$ & $91.21 \pm 1.8$ \\
PB & $11.37 \pm 1.9$ & $22.75 \pm 0.9$ & $25.88 \pm 1.3$ & $28.18 \pm 0.8$ & $29.14 \pm 1.9$ \\
PC & - & $8.93 \pm 1.4$ & $10.20 \pm 1.3$ & $11.31 \pm 0.9$ & $13.24 \pm 0.3$ \\
PV & - & $13.82 \pm 0.9$ & $15.68 \pm 0.6$ & $16.87 \pm 0.9$ & $15.90 \pm 1.7$ \\
CC & $33.45 \pm 2.3$ & $66.88 \pm 4.3$ & $67.57 \pm 4.9$ & $70.29 \pm 5.7$ & $70.46 \pm 4.3$ \\
FDN & $66.55 \pm 2.3$ & $33.12 \pm 4.3$ & $32.43 \pm 4.9$ & $29.71 \pm 5.7$ & $29.54 \pm 4.3$ \\
FDA & $51.01 \pm 4.7$ & $18.04 \pm 3.7$ & $20.05 \pm 5.8$ & $15.95 \pm 4.7$ & $15.30 \pm 3.7$ \\
Hemicelulosa & $15.54 \pm 5.7$ & $15.09 \pm 2.6$ & $12.38 \pm 2.3$ & $13.76 \pm 1.2$ & $14.24 \pm 2.4$ \\
\hline MS: Materia seca, MO: Materia orgánica, PB: Proteína bruta, PC $=$ NNP*6.25, NNP: Nitrógeno no proteico,
\end{tabular}

PV: Proteína verdadera, CC: Contenido celular, FDN: Fibra detergente neutro, FDA: Fibra detergente ácido.

los reportados por Ramos et al. (2006). Para el $\mathrm{pH}$, se encontraron diferencias $(\mathrm{p}<0.0001)$ en las fuentes de variación: animal, período, tiempo de incubación, y tratamientos $(P=0.0494)$, pero no se encontraron para tratamientos por tiempo de incubación, con diferencias entre sF y SP al $0.05 \%$ de urea, de 6.76 y 6.39 , respectivamente $(p<0.05)$. En todos los tratamientos y horarios evaluados, la suplementación con SP mantuvo un pH en los niveles recomendados durante la celulolísis ruminal en pastos y forrajes (Calsamiglia et al. 2008).

Para el $\mathrm{N}-\mathrm{NH}_{3}$, se encontraron diferencias ( $\mathrm{p}$ $<0.0001$ ) en las fuentes de variación: animal, periodo, tratamientos, y en tratamientos por horas de incubación, siendo similar a las 0 horas. Las concentraciones de $\mathrm{N}-\mathrm{NH}_{3}$, a las $2 \mathrm{~h}$ de incubación, en el rumen de los animales que consumieron $\mathrm{sF}+\mathrm{SP}$, se encontraban ligeramente arriba del rango mínimo limitante para una degradación y consumo adecuado (Gutiérrez 2015). Pero en todos los horarios, la concentración de $\mathrm{N}-\mathrm{NH}_{3}$ en $\mathrm{sF}$ fue menor a $8 \mathrm{mg} \mathrm{dL}^{-1}$, a pesar de que el pasto tuvo valores de $\mathrm{PB}$ similares a pastos de buena calidad.

Los estudios de la degradación de la MS, FDN y FDA, tanto in situ (DI), como la DE, son importantes ya que con ellos se puede estudiar el efecto que tiene la suplementación sobre el aprovechamiento de la MS y la fibra de los pastos. Con la DI, se evalúa el proceso de degradación de la biomasa del pasto, y con la DE, el efecto del pasaje en la degradación ruminal. Por lo que la relevancia del modelo desarrollado no radica en la descripción de la cinética de degradación, sino en que permite evaluar la DI y DE al mismo tiempo, y comprender el mecanismo por el cual se da el comportamiento de la DE, por efecto de la concentración de la urea (Rosero y Posada 2017). En la Tabla 3 se observa que la mayor DEMS se dió en la dieta con $1.0 \%$, seguido del tratamiento con $0.5 \%$ de urea, lo cual no se puede explicar únicamente con el comportamiento de la DEFDN, ya que su valor más alto se presentó al $2 \%$ de urea. Al simularse la DEMS se observó un aumentó aproximado de $10 \mathrm{~g}$ del material disponible para el animal por cada $100 \mathrm{~g}$ de forraje, cuando este fue adicionado con $0.5 \%$ ( 0.410536 $0.318358)$ y $1.0 \%(0.410793-0.318358)$ de urea, disminuyendo de 8.5 a $3 \mathrm{~g}$ de aumento por $100 \mathrm{~g}$ de forraje disponible para el SP al 1.5\% (0.403787 - 0.318358$)$ y $2.0 \%$ de urea $(0.347454-0.318358)$, respectivamente. Para la DEFDN se encontró el nivel más alto con el $2.0 \%$ de urea $(0.226014$ 0.135127), aproximadamente por $9 \mathrm{~g}$ por $100 \mathrm{~g}$ de forraje disponible, presentando los tratamientos con $0.5 \%$ (0.19484 - 0.135127), 1.0\% (0.192744 $0.135127)$, y $1.5 \%$ de urea $(0.199105-0.135127)$, menor material disponible, aproximadamente por 6 $\mathrm{g}$ por $100 \mathrm{~g}$ de forraje. Para la DEFDA se observó el valor más alto con el SP de $1.5 \%$ de urea (0.23201 0.098913 ), con un aumento aproximado de $13 \mathrm{~g}$ por $100 \mathrm{~g}$ de forraje de material disponible, presentando los tratamientos con 0.5\% (0.208561 - 0.098913), $1.0 \%(0.201897-0.098913)$ y $2.0 \%(0.196778-$ 
Tabla 3. Degradación Efectiva y cinética de la degradación in situ de la MS, FDN y FDA del pasto Elefante para diferentes niveles de urea en el suplemento, Sacchapulido.

\begin{tabular}{|c|c|c|c|c|c|}
\hline \multirow{2}{*}{ Parámetro } & \multicolumn{5}{|c|}{ Tratamientos } \\
\hline & Forraje & SP con $0.5 \% \mathrm{U}$ & SP con $1.0 \% \mathrm{U}$ & SP con $1.5 \% \mathrm{U}$ & SP con $2.0 \% \mathrm{U}$ \\
\hline \multicolumn{6}{|l|}{ MS } \\
\hline DEMS & 5.89 & 7.48 & 7.63 & 7.24 & 6.18 \\
\hline$k d$ & 0.10 & 0.07 & 0.09 & 0.09 & 0.05 \\
\hline PD & 7.66 & 10,69 & 10.18 & 9.66 & 9.90 \\
\hline ND & 10.85 & 7.53 & 8.40 & 8.27 & 7.88 \\
\hline $\mathrm{EE} \pm$ & 11.17 & 10.32 & 10.87 & 12.59 & 12.48 \\
\hline \multicolumn{6}{|l|}{ FDN } \\
\hline DEFDN & 2.5 & 3.55 & 3.58 & 3.57 & 4.02 \\
\hline$k d$ & 0.11 & 0.17 & 0.15 & 0.11 & 0.12 \\
\hline PD & 3.17 & 4.18 & 4.31 & 4.54 & 5.02 \\
\hline ND & 10.15 & 9.65 & 9.35 & 8.83 & 8.51 \\
\hline $\mathrm{EE} \pm$ & 0.76 & 0.40 & 0.33 & 0.35 & 0.34 \\
\hline \multicolumn{6}{|l|}{ FDA } \\
\hline DEFDA & 1.83 & 3.8 & 3.75 & 4.16 & 3.5 \\
\hline$k d$ & 0.10 & 0.17 & 0.13 & 0.14 & 0.13 \\
\hline PD & 2.36 & 4.47 & 4.62 & 5.08 & 4.30 \\
\hline ND & 5.88 & 4.75 & 4.51 & 3.94 & 3.99 \\
\hline $\mathrm{EE} \pm$ & 0.52 & 0.39 & 0.69 & 0.53 & 0.74 \\
\hline $\begin{array}{l}\text { Sacch } \\
(\mathrm{F}+\end{array}$ & $(1$ & Urea, $k d$ : & $\begin{array}{l}\text { asa de degrada } \\
\text { Error estándar. } \\
\text { (F + SP } 1.0 \%\end{array}$ & $\begin{array}{l}\text { ón, PD: Sustra } \\
\text { Tratamientos: } \\
\text { J), } 1.5 \%(\mathrm{~F}+\end{array}$ & $\begin{array}{l}\text { potencialmente } \\
\text { olo forraje (F), } \\
1.5 \% \text { y } 2.0 \%\end{array}$ \\
\hline
\end{tabular}

0.098913), con un aumento aproximado de $10 \mathrm{~g}$ por $100 \mathrm{~g}$ de forraje. Es importante resaltar que la FDA es la que mayores cambios tuvo con respecto al incremento de la disponibilidad de carbohidratos para la nutrición del animal.

En la Tabla 3 se observa que, para la MS el tratamiento con $0.5 \%$ de urea fue el que tuvo el valor más alto de PD y, por consiguiente, el más bajo de ND (10.69 y $7.53 \%)$, pero el $k d$ se mantuvo relativamente constante $(0.05$ a 0.10$)$. Para la FDN, se observa que la PD y ND tuvieron los valores más altos y más bajos con el $2.0 \%$ de urea (5.02 y $8.51 \%)$. Las $k d$ para la FDN fueron mayores con $0.5(0.17)$ y $1.0 \%(0.15)$ de urea. Para la FDA, se observó el valor más alto de PD en el SP con $1.5 \%$ de urea $(5.08 \%)$ y los valores más bajos de ND en el SP con 1.5 (3.94\%) y 2\% (3.99\%) de urea. Se observa que la $k d$ más alta de la FDA fue para el SP con $0.5 \%$ de urea (0.17) y la más baja para el sF (0.10). Al revisar los valores de los parámetros cinéticos, se observa que en la mayoría de los casos, donde la DE es mayor, también, se observa mayor fracción de PD, pero no de $k d$; por lo que, el tamaño de la fracción PD, y no la tasa de degradación del forraje, es la que se favorece con el $\mathrm{sF}+\mathrm{SP}$ en las dietas. El aumento de la fracción PD podría estar relacionado con la composición de la población bacteriana favorecida por un ambiente ruminal estable y rico en nutrientes (Galindo et al. 1995) como el aportado por las dietas de SP con urea. Los incrementos en la fracción PD pudieron deberse al efecto del aporte de proteína y energía degradable en el rumen, maximizando la actividad microbiana, ya que, de acuerdo con Wang et al. (2017) los carbohidratos disponibles de la dieta son usados como fuentes de energía para el crecimiento de los microorganismos, los cuales, son responsables de la celulolísis ruminal mediante la secreción de enzimas $\beta$ 1-4 glucosidasas. El aumento de la PD y no de la $k d$ puede indicar, más que a un aumento del crecimiento microbiano, relacionado a la $k d$, a un cambio en la composición de la población ruminal. El cambio podría darse, tanto por el incremento de la energía, como del cambio en el tipo de suplementación (Galindo et al. 1995), ambos, relacionados con el incremento en el tipo de compuestos potencialmente degradables por la población microbiana. 

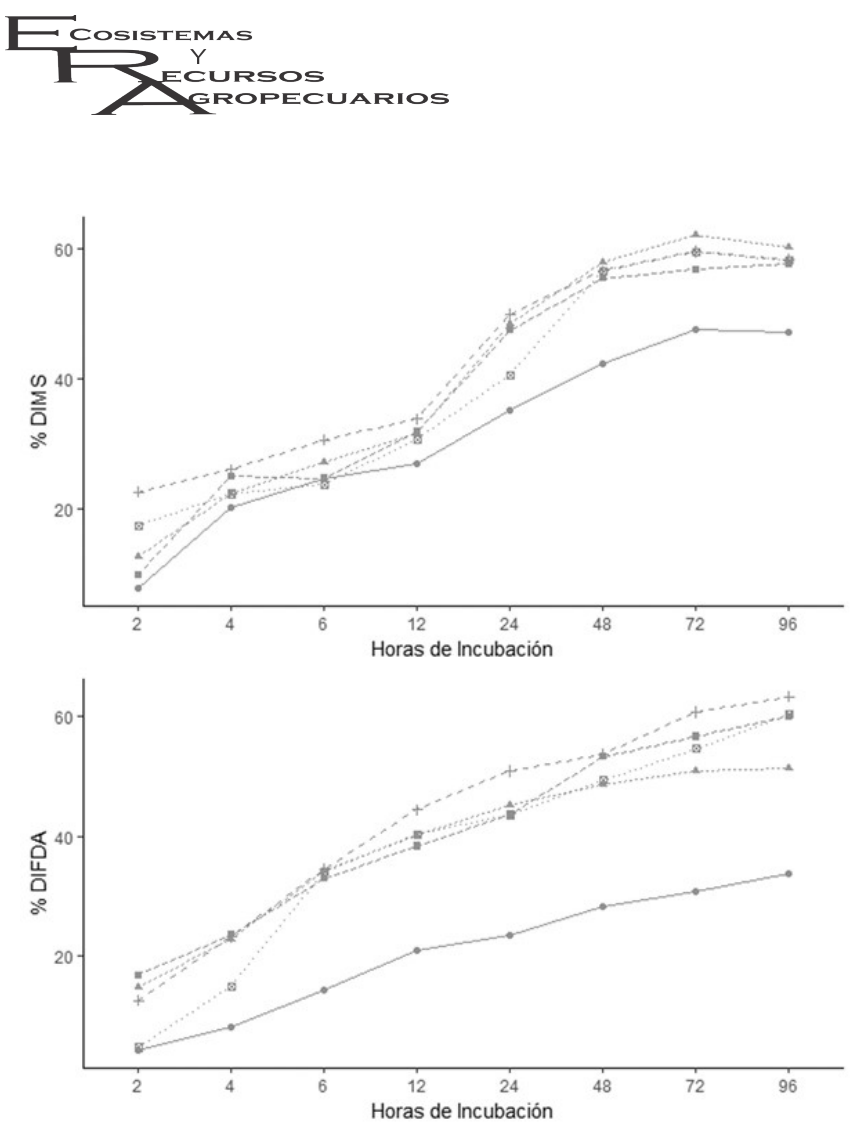

Nivel de urea en el Sacchapulido

Ecosist. Recur. Agropec.

$5(15): 563-571,2018$
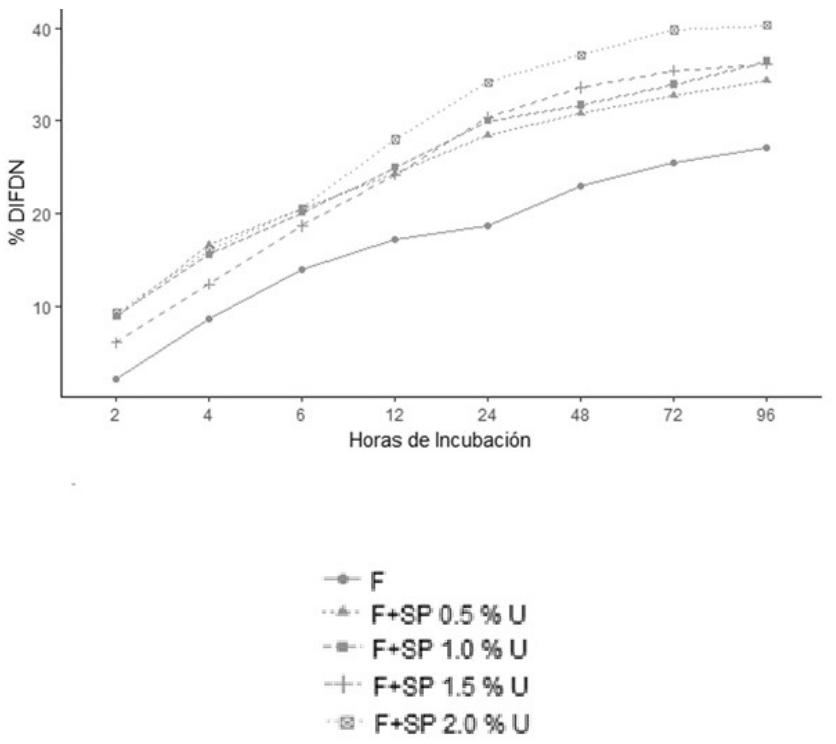

Figura 1. Efecto de niveles de urea en el Sacchapulido sobre la digestibilidad in situ de la MS (DIMS), FDN (DIFDN) y FDA (DIFDA) del forraje Elefante.

La evaluación de las diferencias entre las fracciones de PD, da una idea de los mecanismos biológicos que relacionan los resultados de la DEMS, DEFDN y DEFDA. Las diferencias son consecuencias de cambios en el PD de CC. Para la CC, el incremento del tratamiento $\mathrm{sF}$ al SP fue del 4.49 a $6.51 \%$ de su MS, mientras que los otros tratamientos no tuvieron incrementos observandose niveles de $5.87,5.12$ y $4.88 \%$, para los tratamientos con 1.0 , 1.5 y $2.0 \%$ de urea, respectivamente. Lo anterior explica la razón de que la DEMS de la dieta al 0.5\% de urea fuera la mayor de todas las dietas, aunque tuviera una DEFDN que no sobresalió entre las otras dietas.

La evaluación de las diferencias entre las fracciones de FDN y FDA no aportaron mucha información. El tratamiento con SP al $2.0 \%$ de urea tuvo el nivel más alto de $\mathrm{Hc}(0.72054 \%)$, lo que pudiera explicar el valor superior de la FDN en comparación con los otros niveles de urea. Sin embargo, los otros valores obtenidos por diferencia fueron negativos. Mientras que el tratamiento sF presentó un nivel de Hc mayor al de $2.0 \%$ de urea $(0.80292 \%)$, que no permite determinar el peso de la Hc en la DEMS. Es importante profundizar en el estudio comparativo entre las cinéticas (PD, ND, $k d$ ) de cada fracción del alimento (MS, FDN, FDA) para comprender los cambios que se dan con los diferentes tipos de alimentos, pero también evaluar las metodologías de estimación de parámetros utilizando multiajustes que estudien de manera integral el comportamiento del sistema (Vargas y Tedeschi 2014).

Los valores de DE y parámetros cinéticos muestran un aumento de la degradación entre sF vs $\mathrm{sF}+\mathrm{SP}$. Los cuales deben ser resultado de los incrementos en los valores de DIMS, DIFDN y DIFDA (Figura 1). Sin embargo, al evaluar las curvas de sF $+\mathrm{SP}$, se observa que solamente pueden explicarse los resultados de DEFDN y FDA con los cambios en DIFDN y DIFDA, pero no de DEMS con DIMS, 
debido a que no existe una curva con valores superiores a las otras durante el tiempo de incubación (96 h) para la DIMS, sino que se intercalan entre los niveles de 0.5 y $1.5 \%$ de urea. Lo cual, explica parcialmente que el nivel más alto de DEMS fue de $0.5 \%$ de urea. Pero para los casos de DIFDN y DIFDA la relación con sus respectivas DE son evidentes, ya que, las curva con valores más altos son las de 2.0 y $1.5 \%$ de urea, para DIFDN y DIFDA, respectivamente. Lo encontrado en el caso de la DIMS remarca la importancia de estudiar el efecto de la tasa de pasaje en los estudios de degradación, ya que a veces no es fácil determinarlo únicamente con el estudio de la DI.

La recomendación del uso de SP se basa en el hecho de que la elaboración del SP se realiza con una tecnología sencilla, que es factible de aplicar en cualquier finca. La DEMS mostró que las dietas de forrajes suplementadas con SP al 0.5 y $1.0 \%$ de urea fueron las que permitieron un mayor aprovechamiento de la MS del forraje, presentando un incremento en la eficiencia del $10 \%$. Se recomienda seguir evaluando este nuevo alimento para conocer mejor la cinética de sus diferentes fracciones y así mejorar el aprovechamiento del pasto.

En general, los niveles de $\mathrm{pH}$ y $\mathrm{N}-\mathrm{NH}_{3}$ evaluados, en este trabajo, fueron adecuados para la degradación del pasto Elefante, así como para el crecimiento microbiano ruminal. La suplementación con SP, en todos los niveles de urea, mejoraron la degradación del pasto Elefante, tanto de la DI como de la DE de la MS, así como de sus fracciones de fibras. La extensión de la degradación de la PD como consecuencia de la utilización de SP fue la que determinó el mejor aprovechamiento del forraje. Las concentraciones de urea al 0.5 y $1.0 \%$ en el suplemento de SP tuvieron mayor efecto en el aprovechamiento de la biomasa en el rumen con respecto a las dietas a base de pasto Elefante; lo que permite recomendar al productor el nivel de $0.5 \%$ de urea en el SP debido al costo de la urea.

\section{AGRADECIMIENTOS}

Al Fondo Mixto de Fomento a la Investigación Científica y Tecnológica CONACYT - Gobierno del Estado de Tabasco por el apoyo al proyecto "Intensificación en la producción de alimentos para animales a través de procesos biotecnológicos que protejan al medio ambiente" Clave TAB-2007-C09-74746. Al CONACYT por la beca otorgada al estudiante Carlos Rubén Fernández-Cabrera durante su formación académica en la maestría Producción Agroalimentaria en el Trópico (PROPAT).

\section{LITERATURA CITADA}

AOAC (2016) Official Methods of Analysis. 20 th Edition. AOAC International. Maryland, USA. 3172p

Calsamiglia S, Cardozo PW, Ferret A, Bach A (2008) Changes in rumen microbial fermentation are due to a combined effect of type of diet and pH. Journal of Animal Science 86: 702-711.

Corea EE, Aguilar JM, Alas NP, Alas EA, Flores JM, Broderick GA (2017) Effects of dietary cowpea (Vigna sinensis) hay and protein level on milk yield, milk composition, $\mathrm{N}$ efficiency and profitability of dairy cows. Animal Feed Science and Technology 226: 48-55.

Cruz-Hernández A, Hernández-Garay A, Vaquera-Huerta H, Chay-Canul A, Enríquez-Quiroz J, RamírezVera S (2017) Componentes morfogenéticos y acumulación del pasto mulato a diferente frecuencia e intensidad de pastoreo. Revista Mexicana de Ciencias Pecuarias 8: 101-109.

Galindo J, Marrero Y, Aranda N, Chongo B (1995) Efecto de la Leucaena leucocephala en la población microbiana ruminal en vacas que consumen forraje de caña de azúcar y King grass. Revista Cubana de Ciencias Agrícolas 29: 45-51.

Gutiérrez O (2015) La fisiología digestiva del rumiante, objeto de investigación en el Instituto de Ciencia Animal durante cincuenta años. Revista Cubana de Ciencia Agrícola 49: 179-188. 
Haile E, Njonge FK, Asgedom G, Gicheha M (2017) Chemical Composition and Nutritive Value of AgroIndustrial By-Products in Ruminant Nutrition. Open Journal of Animal Sciences 7: 8-18.

Hernández D, Carballo M, Reyes F (2000) Reflexiones sobre el uso de los pastos en la producción sostenible de leche y carne de res en el trópico. Pastos y Forrajes 23: 269-274.

Kirk RS, Sawyer R, Egan H (2011) Composición y análisis de alimentos de Pearson. $2^{a}$ Edición. Grupo Edit Patria. Distrito Federal, México. 787p.

Lourenço MSN, Messana JD, de O Sader AP, Canesin RC, Malheiros EB, Castagnino PS, et al. (2017) Comparison of laboratory methods for quantification of fiber in feedstuffs. Revista Colombiana de Ciencias Pecuarias 30: 21-29.

Macey R, Oster G, Zahnley T (2003) Berkeley Madonna. User guide version 8.0. University of California, Department of Molecular and Cellular Biology, Berkeley, CA. http://www. berkeleymadonna.com. Fecha de consulta: 27 de junio del 2017.

Montgomery DC (2012) Design and Analysis of Experiments. $8^{\text {th }}$ edition. Wiley. New York, USA. 724p.

Pardo O, Carulla JE, Hess HD (2008) Efecto de la relación proteína y energía sobre los niveles de amonio ruminal y nitrógeno ureico en sangre y leche, de vacas doble propósito del Piedemonte llanero, Colombia. Revista Colombiana de Ciencias Pecuarias 21: 387-397.

Ramos-Juárez JA, Aranda-Ibáñez EM, Morales-Jimenez E (2016) Alimento fermentado a base de caña de azúcar (Saccharum spp.) en el cambio de peso de bovinos en pastoreo. Agroproductividad 9: 56-61.

Ramos JA, Elías A, Herrera F (2006) Procesos para la producción de un alimento energético - proteico para animales. Efecto de cuatro fuentes energéticas en la fermentación en estado sólido (FES) de la caña de azúcar. Revista Cubana de Ciencia Agrícolas 40: 51-58.

Reid M, O'Donovan M, Elliott CT, Bailey JS, Watson CJ, Lalor STJ, et al. (2015) The effect of dietary crude protein and phosphorus on grass-fed dairy cow production, nutrient status, and milk heat stability. Journal of Dairy Science 98: 517-531.

Rosero R, Posada SL (2007) Modelación de la cinética de degradación de alimentos para rumiantes. Revista Colombiana de Ciencias Pecuarias 20: 174-182.

Rusdy M (2016) Elephant grass as forage for ruminant animals. Livestock Research for Rural Development 28, Artículo 49. http://www.Irrd.org/lrrd28/4/rusd28049.html. Fecha de consulta: 27 de junio de 2017.

SAS (2017) Base SAS ${ }^{\circledR}$ 9.4 Procedures guide. 7th Edition. SAS ${ }^{\circledR}$ Documentation. SAS Institute Inc. https://support.sas.com/documentation/cdl/en/proc/70377/PDF/default/proc.pdf. Fecha de consulta: 3 de julio de 2017.

Sosa D, Dustet JC, Boucourt R, Rodríguez Z (2016) Validation of a mathematical model developed for the solid state fermentation process of the sugar cane (Saccharum officinarum) with sweet potato (Ipomoea batata Lam). Revista Cubana de Ciencia Agrícola 50: 25-38.

Uddin MJ, Khandaker ZH, Khan MJ, Khan MMH (2015) Dynamics of microbial protein synthesis in the rumen: A Review. Annals of Veterinary and Animal Science 2: 116-131.

Vargas-Villamil LM, Tedeschi LO (2014) Potential integration of multi-fitting, inverse problem and mechanistic modelling approaches to applied research in animal science: a review. Animal Production Science. 54: 1905-1913. 
Wang Y, Cao P, Wang L, Zhao Z, Chen Y, Yang Y (2017) Bacterial community diversity associated with different levels of dietary nutrition in the rumen of sheep. Applied Microbiology and Biotechnology 101: 3717-3728. 
